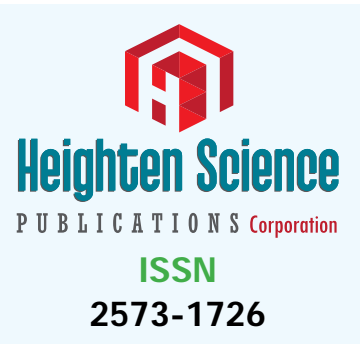

*Address for Correspondence: Antonio Eduardo de Aquino J r., Ph.D. Vanderlei Salvador Bagnato, Ph.D, São Carlos Institute of Physics, University of São Paulo, PO Box 369, 13560-970,

São Carlos, São Paulo, Brazil, Tel: +55 (16) 3373 9810; Email: antoniodeaquinojr@gmail.com; vander@ifsc.usp.br

Submitted: 18 J anuary 2018

Approved: 23 J anuary 2018

Published: 24 J anuary 2018

Copyright: (c) 2018 J orge AES, et al. This is an open access article distributed under the Creative Commons Attribution License, which permits unrestricted use, distribution, and reproduction in any medium, provided the original work is properly cited.

Keywords: Hand osteoarthritis; Physical therapy Low-level laser therapy; Ultrasound therapy
Case Report

\section{Ultrasound conjugated with Laser Therapy in treatment of osteoarthritis: A case study}

\author{
Ana Elisa Serafim Jorge ${ }^{1}$, Michele Luise de Souza Simão ${ }^{2}$, Ana \\ Carolina Fernades ${ }^{2}$, Aline Chiari ${ }^{2}$, Antonio Eduardo de Aquino \\ Junior ${ }^{2 *}$, Anderson Luis Zanchin ${ }^{3}$ and Vanderlei Salvador \\ Bagnato ${ }^{2 *}$ \\ ${ }^{1}$ Federal University of São Carlos, São Carlos, São Paulo, Brazil \\ ${ }^{2}$ Sao Carlos Institute of Physics, University of São Paulo, São Carlos, São Paulo, Brazil \\ ${ }^{3}$ MMOptics, São Carlos, São Paulo, Brazil
}

\section{Abstract}

Osteoarthritis of the hand is a chronic condition that involves hand joints, but receives less attention. Few studies have investigated the use of ultrasound therapy and laser therapy for the treatment of hand osteoarthritis. The objective was to evaluate the effect of the conjugated treatment of therapeutic ultrasound and laser therapy on the pain and joint function of a patient with hand osteoarthritis. The is case of a woman, 57 years old, with a diagnosis of osteoarthritis on hand for 3 years, presenting constant pain and worsening after manual activities. The pain and function were evaluated, respectively, by Visual Analog Scale (VAS) and Australian Canadian Osteoarthritis Hand Index questionnaire (AUSCAN). After 12 sessions using ultrasound and laser therapy application, there was an expressive improvement in the pain and functional indexes of the patient. The combined application of therapeutic ultrasound and laser therapy, through the unified field action of the therapies used, proved to be efficient in reducing pain and improving the functionality.

\section{Introduction}

Osteoarthritis (OA) of the hand is a chronic condition that involves one or more joints of the hand associated with pain, joint limitation, loss of strength, swelling and functional disability in daily activities. There is an estimate that reaches about $26 \%$ of people, mostly women $[1,2]$.

According to the European League Against Rheumatism (EULAR), the treatment for osteoarthritis of the hand include medicines, exercise, use of orthoses, electrotherapy, thermotherapy, massage and acupuncture for improvement of symptoms. However, laser therapy and ultrasound therapy are poorly used, although they have great therapeutic potential [1-3]. Laser therapy is known as a photobiomodulator which has been used as a non-invasive treatment in osteoarthritis for pain relief. The studies are based on the anti-inflammatory effect of light as an explanation for the improvement of pain $[1,2,4]$.

In addition to laser therapy, other studies show the benefits of ultrasound (US) in improving pain and function of pathologies such as carpal tunnel syndrome. However, there are few studies in patients with hand osteoarthrosis $[5,6]$. In this context, the use of technology as therapy proves to be an excellent treatment option, due to the need to decrease medication intake.

How to cite this article: J orge AES, Simão MLD, Fernades AC, Chiari A, de Aquino I $r$, AE. et al. Ultrasound conjugated with Laser Therapy in treatment of osteoarthritis: A case study.J Sports Med Ther. 2018; 3: 024-027. https://doi.org/10.29328/journal.jsmt.1001023 
The aim of this brief report is to illustrate the effect of a combined therapeutic ultrasound and laser therapy treatment for symptomatic hand osteoarthritis (OA). Besides, the prototype of equipment was specially developed to promote the conjugated application (US and Laser), which can become a differential in treatment.

\section{Case Report}

A woman at 57 years old, Caucasian, was admitted to our Unit of Photodynamic Therapy of Santa Casa de São Carlos, São Paulo, Brazil, complaining of constant pain in the right hand with diagnosis of hand osteoarthritis for 3 years. In the initial evaluation she presented constant pain in the hand with worsening in the manual activities, like piano, in addition to local heat and morning stiffness.

We collected information about her health status, including age, time frame of osteoarthritis diagnosis, comorbidities, and log of medication. We analyzed some important outcome variables, e.g. pain and physical function, which were assessed by a visual analogue scale (VAS) and Australian Canadian Osteoarthritis Hand Index (AUSCAN), performed before and after the treatment. The function was evaluated through the block test performed in 1 minute.

\section{Equipment and Intervention}

It was used a prototype developed by Laboratory of Technological Support of the Institute of Physics of São Carlos, University of São Paulo (USP). This specific equipment promotes the conjugated therapy between ultrasound and laser therapy, allowing the action of therapeutic field overlap with laser and ultrasound (Figure 1). The combined therapy was applied to both hands, 2 times per week, during 12 sessions. The application protocol was performed in the palmar region of the patient's hand. The probe was kept in circular constant movements (angle of $90^{\circ}$ with the skin surface). It was used gel and total contact in order to ensure an optimal energy delivery. The time of application was 12 minutes for each hand $[7,8]$.

\section{Results and Discussion}

Considering the evidences presented in relation to ultrasound and laser therapy as non-pharmacological forms of treatment, this case study points to an expressive improvement in pain and function indexes in a patient with osteoarthritis in hand after treatment with innovative equipment that conjugates the application of therapeutic ultrasound with laser therapy, generating field overlap. Table 1 shows the data computed in the initial and final phases of the treatment. It is possible to observe an expressive improvement in the pain index evaluated by both the VAS and the AUSCAN questionnaire.

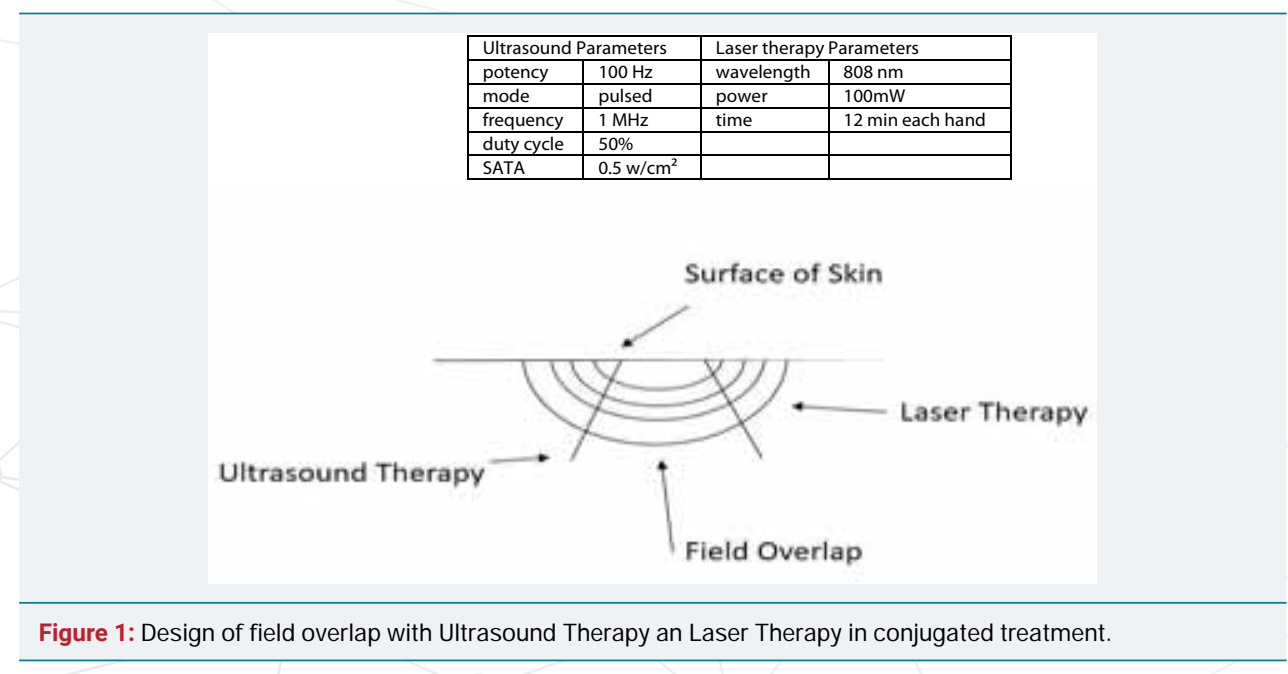


Table 1: Initial and Final data of variables of Visual Analogue Scale (VAS), Australian Canadian Osteoarthritis Hand Index (AUSCAN).

\begin{tabular}{|c|c|c|c|}
\hline Outcomes & \multicolumn{2}{c|}{ Initial } \\
\hline \multicolumn{2}{|c|}{ VAS } \\
\hline Pain & 9.8 & 1.2 & -716.6 \\
\hline Pain & AUSCAN & & \\
\hline J oint Stiffness & 10 & 3 & -233 \\
\hline Daily Living Activity & 1 & 0 & 0 \\
\hline Total & 3 & 3 & 0 \\
\hline AUSCAN-Australian Canadian Osteoarthritis Hand Index; VAS-Visual Analogue Scale. & 6 & -133.3 \\
\hline
\end{tabular}

There are few evidences found in the literature using these therapeutic modalities in hand osteoarthritis. Studies have shown the efficacy of therapeutic ultrasound in individuals with knee osteoarthritis by up to $21 \%$ in pain improvement using the device in pulsed mode and low intensity $[9,10]$. Another non-invasive therapeutical modality that has been used for several years in the control of pain is laser therapy. Its anti-inflammatory effect is pointed out in several studies [1,11,12]. However, in this case study where the therapy used was conjugated, VAS presented a percentage decrease of $-716.66 \%$ in relation to pain perception. The Auscan questionnaire presented a significant decrease for pain quantification of $-233.33 \%$ (Table 1). These results are corroborated by recent studies published by our group, which points out a great improvement in pain questionnaires for knee and hand osteoarthritis [7,8]. In addition to such significance, there was a return to the activity of playing the piano, without any sign of pain or limitation. In this way, our results corroborate the results of singular treatments found in the literature $[7,8]$. However, the results obtained make this model of equipment and therapy potentially used more beneficial for the treatment of osteoarthritis.

\section{Conclusion}

This case shows the effectiveness of the conjugated therapy with ultrasound and laser therapy. The results shown apoint that the technological treatment of hand osteoarthritis is efficient, being able to discard options of pharmacological and invasive treatment. In this way, the use of new technologies through the efficiency of field overlap in this equipment, as well as a new methodology in the treatment of osteoarthritis, are more viable to better the life quality.

\section{Ethical Approval}

The study was approved by the Hospital Ethical Committe (resolução 466/2012).

\section{Funding}

This work was supported in part by CNPq (INOF-INCT grant 573587/2008-6); FINEP (grant 01.13.0430-00) and São Paulo Research Foundation (FAPESP) grant 2013/07276-1 (CePOF).

\section{References}

1. Baltzer AWA, Ostapeczuk MS. Positive Effects of Low Level Laser Therapy (LLLT) on Bouchard's and Heberden's Osteoarthritis. Lasers Surg Med. 2016; 48: 498-504. Ref.: https://goo.gl/ccCwVW

2. Ye L, Kalichman L, Spittle A, Dobson F, Bennell K. Effects of rehabilitative interventions on pain, function and physical impairments in people with hand osteoarthritis: a systematic review. Arthritis Research \& Therapy. 2011; 13: 28. Ref.: https://goo.gl/Cq1ojU

3. Beasley J . Osteoarthritis and Rheumatoid Arthritis: Conservative Therapeutic Management. J Hand Ther. 2012; 25: 163-172. Ref.: https://goo.gl/b4MsNa

4. Brosseau L, Wells G, Marchand S, Gaboury I, Stokes B, et al. Randomized controlled trial on low level laser therapy (LLLT) in the treatment of osteoarthritis (OA) of the hand. Lasers Surg Med. 2005; 36: 210-219. Ref.: https://goo.gl/W486Uc 
5. Page MJ , O'Connor D, Pitt V, Massy-Westropp N. Therapeutic ultrasound for carpal tunnel syndrome. Cochrane Database of Systematic Reviews. 2013; 3. Ref.: https://goo.gl/HPLtFY

6. Kortekass MC, Kwok WY, Reijnierse M, Stijnen T, Kloppenburg M. Brief Report: Association of inflammation with development of erosions in patientes with hand osteoarthritis: A Prospective ultrasonography study. Arthitis Rheumatol. 2016; 68: 392-397. Ref.: https://goo.gl/NrHZUw

7. J orge AES, Simão MLS, Fernandes AC, Chiari A, de Aquino J r AE, et al. Can combined ultrasound and laser therapy potentiate the treatment of a symptomatic osteoarthritis? A case report. J ournal of Novel Physioterapies. 2017; 7: 1-3.

8. Paolillo AR, Paolillo FR, J oão J P, J oão HA, Bagnato VS. Synergic effects of ultrasound and laser on pain relief in women hand osteoarthritis. Lasers in Medical Science. 2015. 30: 279-286. Ref.: https://goo.gl/CXcaXj

9. Loyola-Sánchez A, Richardson J , MacIntyre NJ . Efficacy of ultrasound therapy for the management of knee osteoarthritis: a systematic review with meta-analysis. Osteoarthritis and Cartilage. 2010; 18: 1117-1126. Ref.: https://goo.gl/k9GZ7G

10. Rodriguez-Merchan EC. Conservative treatment of acute knee osteoarthritis: A review of the Cochrane Library. J ournal of Acute Disease. 2016; 5: 190-193. Ref.: https://goo.gl/xT6Pp6

11. J ang $\mathrm{H}$, Lee $\mathrm{H}$. Meta-Analysis of pain relief effects by laser irradiation on joint áreas. Photomedicine and Laser Surgery. 2012; 30: 1-13. Ref.: https://goo.gl/gHjni6

12. Alfredo PP, Bjordal J M, Steagall J unior W, et al. Long-term results of a randomized,controlled, doubleblind study of low-level laser therapy before exercises in knee osteoarthritis: laser and exercises in knee osteoarthritis. Clinical Rehabilitation. 2017; 1-6. 